\title{
Discriminating Between Exponential and Lindley Distributions
}

\author{
V. S. Vaidyanathan*, Sharon Varghese A \\ Department of Statistics, Pondicherry University, Puducherry, India
}

\section{ARTICLE INFO}

\section{Article History}

Received 11 Oct 2017

Accepted 05 June 2018

\section{Keywords}

Hellinger distance

Lindley distribution

Meijer G-function

Probability of correct selection

Pseudo

Likelihood estimator

Ratio of maximum likelihoods

\section{ABSTRACT}

In literature, Lindley distribution is considered as an alternate to the exponential distribution. In the present work, a methodology is developed to discriminate between exponential and Lindley distributions based on the ratio of the maximum likelihoods. Asymptotic distribution of the test statistic under the null hypothesis is derived and the minimum sample size required to discriminate between the two distributions for a user specified probability of correct selection is obtained. Numerical illustrations of the methodology are given through simulated and real life data sets.

(C) 2019 The Authors. Published by Atlantis Press SARL. This is an open access article distributed under the CC BY-NC 4.0 license (http://creativecommons.org/licenses/by-nc/4.0/).

\section{INTRODUCTION}

In early days of research in reliability theory, exponential distribution is used widely to model failure time data because of its analytical simplicity. However, constant failure rate property of exponential distribution is not always suitable in most of the modern reliability engineering problems. Therefore, many life time distributions have been proposed and studied in literature as an alternative to exponential distribution to model lifetime data. Lindley [1] proposed a probability distribution to show that Bayes' distribution and fiducial distribution are not always same. Ghitany et al. [2] studied various characteristics of Lindley distribution and has shown that Lindley distribution can be used as a better alternative to exponential distribution. Cumulative distribution function (c.d.f) and probability density function (p.d.f) of Lindley distribution with parameter $\beta$, denoted by $\operatorname{Li}(\beta)$, is given by

$$
\begin{aligned}
& F(x)=1-\frac{1+\beta+\beta x}{1+\beta} e^{-\beta x}, x \geq 0, \beta>0 \\
& f(x)=\frac{\beta^{2}}{1+\beta}(1+x) e^{-\beta x}, x \geq 0, \beta>0 .
\end{aligned}
$$

Some generalizations and modifications of Lindley distribution have been suggested by Zakerzadeh and Dolati [3], Nadarajah et al. [4], Bakouch et al. [5], Vaidyanathan and Sharon Varghese [6]. Krishna and Kumar [7], Mazucheli and Achcar [8], Al-Mutairi et al. [9] have studied applications of Lindley distribution in analyzing lifetime data.

From (2), it is clear that the p.d.f of $\operatorname{Li}(\beta)$ is a mixture of exponential $(\beta)$ and $\operatorname{Gamma}(\beta, 2)$ with respective weights $\beta /(1+\beta)$ and $1 /(1+\beta)$. Thus for larger values of $\beta$ both the Lindley and exponential distributions can fit the underlying data reasonably well. However, other life time characteristics such as hazard rate function, mean time to failure, etc., are different for these distributions. From a statistical modelling perspective, it is of interest to know which distribution is more appropriate for the given data. This serves as a motivation to develop a discrimination procedure to discriminate between exponential and Lindley distributions using the sample data. In literature, many authors have considered ratio of maximum likelihoods (RML) to discriminate between various distributions. RML is used by Cox [10] and 
Cox [11] for discriminating between two non-nested models. Dumonceaux and Antle [12] have used RML to discriminate between lognormal and Weibull distributions. A procedure for discriminating between Weibull and log-normal distributions is developed by Kundu and Manglick [13]. Kundu et al. [14] have considered discriminating between log-normal and generalized exponential distributions. To the best of authors' knowledge, no work has been done in discriminating between exponential and Lindley distributions. Thus the aim of this paper is to develop a discrimination procedure based on RML to discriminate between exponential and Lindley distributions. Rest of the paper is organized as follows. In Section 2, discrimination procedure based on RML is developed and the probability of correct selection (PCS) of the procedure is defined. Section 3 discusses asymptotic properties of the RML under null hypotheses. In Section 4, proposed methodology is validated using simulation study. Section 5 deals with the numerical illustration of the developed procedure using two real life data sets. A procedure to determine the minimum sample size required to discriminate between the two distributions is proposed in Section 6. Conclusion of the paper is given in Section 7.

\section{DISCRIMINATION PROCEDURE}

In this section, likelihood ratio test statistic for testing whether the given data is from the exponential distribution (exp $(\alpha))$ with p.d.f

$$
f(x)=\alpha e^{-\alpha x}, x \geq 0, \alpha>0
$$

or Lindley distribution $(\operatorname{Li}(\beta))$ is derived. Suppose $x_{1}, x_{2}, \ldots, x_{n}$ is a random sample of $n$ observations from a population having distribution either $\exp (\alpha)$ or $\operatorname{Li}(\beta)$. An experimenter wishes to know from which distribution the given data has arisen. For this, we consider the RML defined as

$$
R M L=\frac{L_{e x p}(\hat{\alpha})}{L_{L i}(\hat{\beta})},
$$

where $L_{\text {exp }}(\hat{\alpha})$ and $L_{L i}(\hat{\beta})$ are likelihood functions corresponding to (3) and (2) respectively and $\hat{\alpha}$ and $\hat{\beta}$ are the corresponding maximum likelihood estimators (MLEs) of $\alpha$ and $\beta$. Substituting respective likelihood functions in (4) and taking natural logarithm on both sides, we get logarithm of RML, denoted by $T$ as

$$
T=n \log (\hat{\alpha})-\hat{\alpha} \sum_{i=1}^{n} x_{i}-2 n \log \hat{\beta}-\sum_{i=1}^{n} \log \left(1+x_{i}\right)+\hat{\beta} \sum_{i=1}^{n} x_{i}+n \log (1+\hat{\beta}),
$$

where $\hat{\alpha}$ and $\hat{\beta}$ are given by

$$
\left.\begin{array}{l}
\hat{\alpha}=\frac{1}{\bar{x}} \\
\hat{\beta}=\frac{-(\bar{x}-1)+\sqrt{(\bar{x}-1)^{2}+8 \bar{x}}}{2 \bar{x}}
\end{array}\right\} .
$$

The proposed procedure is as follows: Select exponential distribution if $T>0$, otherwise select Lindley distribution. $T>0$ implies that numerator of RML is larger than the denominator. This means that the sample observations are more likely to have been generated from exponential distribution than from Lindley distribution and hence we choose $\exp (\alpha)$ to model the given data. If $T<0$, then denominator of RML will be larger than the numerator, which implies $\operatorname{Li}(\beta)$ is a better fit to the data.

To get an insight into the performance of the discrimination procedure, PCS is used. PCS, like the notion of power in hypothesis testing, gives the probability of selecting the null distribution under consideration. In the current context, PCS is defined as

$$
P_{\exp (L i)}\left(L_{\exp }(\hat{\alpha})>(<) L_{L i}(\hat{\beta}) \mid \text { data is from } \exp (\alpha)(\operatorname{Li}(\beta))\right)
$$

In terms of $T$, PCS can be defined as

$$
P_{\exp }(T>0)=P(T>0 \mid \text { data is from } \exp (\alpha)) \text {. }
$$

Similarly, in the case of Lindley distribution, PCS is given as

$$
P_{L i}(T<0)=P(T<0 \mid \text { data is from } \operatorname{Li}(\beta)) .
$$




\section{ASYMPTOTIC PROPERTIES OF THE RML UNDER NULL HYPOTHESES}

In this section, asymptotic distribution of the logarithm of RML under null hypotheses is obtained under two different cases.

Case 1: The null hypothesis is that given data is from $\exp (\alpha)$ and the alternative is that given data is from $\operatorname{Li}(\beta)$. The following lemma is helpful in finding the asymptotic distribution of $\mathrm{T}$.

Lemma 1. Under the assumption that data is from $\exp (\alpha)$, as $n \rightarrow \infty$

a. $\hat{\alpha} \rightarrow \alpha$ a.s.

b. $\hat{\beta} \rightarrow \beta^{*}$ a.s, where $\beta^{*}$ is that value of $\beta$ which maximizes $E_{\exp }\left(\log f_{L i}(x ; \beta)\right)$.

c. $\quad$ Let $T^{*}=\log \left(L_{\text {exp }}(\alpha) / L_{L i}\left(\beta^{*}\right)\right)$. Then $\left(T-E_{\text {exp }}(T)\right) / \sqrt{n}$ is asymptotically equivalent to $\left(T^{*}-E_{\text {exp }}\left(T^{*}\right)\right) / \sqrt{n}$.

\section{Proof.}

a. Using the convergence property of MLE, it is clear that under null hypothesis $\hat{\alpha} \rightarrow \alpha$ a.s.

b. Under the null hypothesis, it is observed that $\hat{\beta}$ is a pseudo MLE of $\beta$. Hence $\hat{\beta} \nrightarrow \beta$. However $\hat{\beta} \rightarrow \beta^{*}$ a.s, where $\beta^{*}$ is the value of $\beta$ that minimizes Kullback-Leibler information criteria. i.e., $\beta^{*}$ is obtained by minimizing

$$
E_{\exp }\left[\log \left(\frac{f_{\exp }(x ; \alpha)}{f_{L i}(x ; \beta)}\right)\right] .
$$

It is clear that minimizing the above expectation is same as maximizing $E_{\exp }\left(\log f_{L i}(x ; \beta)\right)$ and calculus approach yields $\beta^{*}$ as

$$
\beta^{*}=\frac{\alpha-1+\sqrt{1+6 \alpha+\alpha^{2}}}{2} .
$$

c. We have to show that $\left[\left(T-E_{\text {exp }}(T)\right) / \sqrt{n}\right]-\left[\left(T^{*}-E_{\text {exp }}\left(T^{*}\right)\right) / \sqrt{n}\right] \rightarrow 0$ in probability in order to show that $\left(T-E_{\text {exp }}(T)\right) / \sqrt{n}$ and $\left(T^{*}-E_{\exp }\left(T^{*}\right)\right) / \sqrt{n}$ are asymptotically equivalent. Since $\hat{\alpha} \rightarrow \alpha$ a.s and $\hat{\beta} \rightarrow \beta^{*}$ a.s, and applying $2 c 4$ XIII in Radhakrishna Rao [15], we get

$$
\begin{aligned}
T & =\log \left(\frac{\prod_{i=1}^{n} f_{\exp }\left(x_{i} ; \hat{\alpha}\right)}{\prod_{i=1}^{n} f_{L i}\left(x_{i} ; \hat{\beta}\right)}\right) \\
& =\sum_{i=1}^{n} \log \left(f_{\exp }\left(x_{i} ; \hat{\alpha}\right)\right)-\sum_{i=1}^{n} \log \left(f_{L i}\left(x_{i} ; \hat{\beta}\right)\right) \\
& \rightarrow \sum_{i=1}^{n} \log \left(f_{\exp }\left(x_{i} ; \alpha\right)\right)-\sum_{i=1}^{n} \log \left(f_{L i}\left(x_{i} ; \beta^{*}\right)\right)=T^{*} \text { in probability. }
\end{aligned}
$$

Also by applying corollary 5.7 in Bartle [16], we get

$$
\begin{aligned}
E(T) & =\int_{x} \log \left(\frac{\prod_{i=1}^{n} f_{\exp }\left(x_{i} ; \hat{\alpha}\right)}{\prod_{i=1}^{n} f_{L i}\left(x_{i} ; \hat{\beta}\right)}\right) d F_{\text {exp }}(x) \\
& \rightarrow \int_{x} \log \left(\frac{\prod_{i=1}^{n} f_{\exp }\left(x_{i} ; \alpha\right)}{\prod_{i=1}^{n} f_{L i}\left(x_{i} ; \beta^{*}\right)}\right) d F_{\text {exp }}(x)=E\left(T^{*}\right) \text { in probability. }
\end{aligned}
$$

Therefore, $\left(T-E_{\exp }(T)\right) / \sqrt{n}$ is asymptotically equivalent to $\left(T^{*}-E_{\exp }\left(T^{*}\right)\right) / \sqrt{n}$.

Theorem 1. Under the assumption that the data is from a exp $(\alpha), T$ is asymptotically distributed as normal with mean $E_{\text {exp }}(T)$ and variance $V_{\text {exp }}(T)$.

Proof. Using central limit theorem, it is observed that $\left(T^{*}-E_{\text {exp }}\left(T^{*}\right)\right) / \sqrt{n}$ is asymptotically distributed as normal with variance $n V_{\text {exp }}\left(T^{*}\right)$. By part $(c)$ of Lemma 1 , it is clear that $\left(T-E_{\text {exp }}(T)\right) / \sqrt{n}$ is asymptotically equivalent to $\left(T^{*}-E_{\text {exp }}\left(T^{*}\right)\right) / \sqrt{n}$. Therefore, $\left(T-E_{\exp }(T)\right) / \sqrt{n}$ is also asymptotically distributed as normal with variance $n V_{\exp }(T)$. 
The expressions for asymptotic mean and variance are obtained as,

$$
\begin{aligned}
\lim _{n \rightarrow \infty} \frac{E_{\text {exp }}(T)}{n}= & \log \alpha-1-2 \log \beta^{*}-e^{\alpha} \Gamma(0, \alpha)+\frac{\beta^{*}}{\alpha}+\log \left(1+\beta^{*}\right), \\
\lim _{n \rightarrow \infty} \frac{V_{\text {exp }}(T)}{n}= & -2\left(\beta^{*}-\alpha\right)\left(G_{2,3}^{3,1}\left(\alpha \mid \begin{array}{c}
-1,0 \\
-1,-1,1
\end{array}\right)-\frac{e^{\alpha} \Gamma(0, \alpha)}{\alpha}\right) \\
& +e^{\alpha}\left(2 G_{2,3}^{3,0}\left(\alpha \mid \begin{array}{c}
1,1 \\
0,0,0
\end{array}\right)-e^{\alpha} \Gamma(0, \alpha)^{2}\right)+\frac{\left(\beta^{*}-\alpha\right)^{2}}{\alpha^{2}}
\end{aligned}
$$

where $G$ and $\Gamma$ denote Meijer G-function and incomplete gamma function respectively. Both these special functions can be evaluated using "gsl" package in R program. Using (10) and (11), asymptotic PCS can be obtained as

$$
P_{\exp }(T>0)=1-\Phi\left(-\frac{E_{\exp }(T)}{\sqrt{V_{\exp }(T)}}\right)
$$

where $\Phi$ denotes c.d.f of the standard normal distribution.

Case 2: The null hypothesis is that given data is from $\operatorname{Li}(\beta)$ and the alternative hypothesis is that given data is from $\exp (\alpha)$. The following lemma is needed to find the asymptotic distribution of $\mathrm{T}$.

Lemma 2. Under the assumption that data is from $\operatorname{Li}(\beta)$, as $n \rightarrow \infty$

a. $\hat{\beta} \rightarrow \beta$ a.s.

b. $\hat{\alpha} \rightarrow \alpha^{*}$ a.s, where $\alpha^{*}$ is that value of $\alpha$ which maximizes $E_{L i}\left(\log f_{\exp }(x ; \alpha)\right)$.

c. $\quad$ Let $T^{*}=\log \left(L_{\text {exp }}\left(\alpha^{*}\right) / L_{L i}(\beta)\right)$. Then $\left(T-E_{L i}(T)\right) / \sqrt{n}$ is asymptotically equivalent to $\left(T^{*}-E_{L i}\left(T^{*}\right)\right) / \sqrt{n}$.

Proof. The proof is similar to that of Lemma 1 with $\alpha^{*}$ given by

$$
\alpha^{*}=\frac{\beta(\beta+1)}{\beta+2} .
$$

Theorem 2. Under the assumption that the data is from a Li( $\beta), T$ is asymptotically distributed as normal with mean $E_{L i}(T)$ and variance $V_{L i}(T)$.

Proof. By central limit theorem, it can be shown that $\left(T^{*}-E_{L i}\left(T^{*}\right)\right) / \sqrt{n}$ is asymptotically distributed as normal with variance $n V_{L i}\left(T^{*}\right)$. By part (c) of Lemma 2, it is clear that $\left(T-E_{L i}(T)\right) / \sqrt{n}$ is asymptotically equivalent to $\left(T^{*}-E_{L i}\left(T^{*}\right)\right) / \sqrt{n}$. Therefore, $\left(T-E_{L i}(T)\right) / \sqrt{n}$ is also asymptotically distributed as normal with variance $n V_{L i}(T)$.

The expressions for asymptotic mean and variance under case 2 are obtained as

$$
\begin{aligned}
\lim _{n \rightarrow \infty} \frac{E_{L i}(T)}{n}= & -\frac{1}{\beta(\beta+1)}\left(G_{2,3}^{3,1}\left(\beta \mid \begin{array}{c}
1,2 \\
1,1,3
\end{array}\right)+\alpha^{*}(\beta+2)\right. \\
& +\beta\left(-\beta \log \left(\alpha^{*}\right)-\log \left(\alpha^{*}\right)-\beta-\beta \log (\beta+1)\right. \\
& \left.\left.+2(\beta+1) \log (\beta)-\log (\beta+1)+e^{\beta} \beta \Gamma(0, \beta)-2\right)\right), \\
\lim _{n \rightarrow \infty} \frac{V_{\text {exp }}(T)}{n}= & \frac{1}{(\beta+1)^{2}}\left(2 ( \beta - \alpha ^ { * } ) \left(\beta ^ { 2 } ( \beta + 1 ) \left(G_{2,3}^{3,1}\left(\beta \mid \begin{array}{c}
-3,-2 \\
-3,-3,0
\end{array}\right)\right.\right.\right. \\
& \left.+G_{2,3}^{3,1}\left(\beta \mid \begin{array}{c}
-2,-1 \\
-2,-2,0
\end{array}\right)\right)-(\beta+2)\left(G_{2,3}^{3,1}\left(\beta \mid \begin{array}{c}
-1,0 \\
-1,-1,1
\end{array}\right)\right. \\
& \left.\left.+e^{\beta} \Gamma(0, \beta)\right)\right)+2 e^{\beta}(\beta+1) G_{3,4}^{4,0}\left(\beta \mid \begin{array}{c}
1,1,1 \\
0,0,0,2
\end{array}\right) \\
& \left.-\left(G_{2,3}^{3,1}\left(\beta \mid \begin{array}{c}
0,1 \\
0,0,2
\end{array}\right)+e^{\beta} \beta \Gamma(0, \beta)\right)^{2}+\frac{\left(\beta^{2}+4 \beta+2\right)\left(\beta-\alpha^{*}\right)^{2}}{\beta^{2}}\right) .
\end{aligned}
$$


Using (14) and (15), asymptotic PCS can be obtained as

$$
P_{L i}(T<0)=\Phi\left(-\frac{E_{L i}(T)}{\sqrt{V_{L i}(T)}}\right) .
$$

\section{SIMULATION STUDY}

This section deals about how asymptotic results work for finite sample sizes using simulated data sets. PCS based on both simulation and asymptotic results is calculated for different choice of parameter values of the null distributions. R programming language version 3.3 .2 is used to perform numerical computations. The algorithm for calculating PCS based on simulation under case 1 is given below.

1. Generate a sample of size $n$ from $\exp (\alpha)$.

2. Compute MLEs for both $\alpha$ and $\beta$ as given in (6).

3. Compute $T$ using (5).

4. Repeat the steps 1-3 for $R$ times and count the number of positive T's.

An estimate of PCS based on simulation can be computed as

$$
\widehat{P C S}=\frac{\text { Number of positive } T^{\prime} s}{R} .
$$

In this study, R is taken to be 1000. PCS computed using simulation and asymptotic results using (12) under case 1 for different choice of parameter values namely, $\alpha=0.2,0.4,0.8,1,1.5,3,6$ and different samples sizes $n=20,40,60,80,100$ is presented in Table 1 . The entries in parenthesis represent asymptotic PCS values.

For case 2, PCS based on simulation can be obtained using the following algorithm.

1. Generate a sample of size $n^{\prime}$ from $\operatorname{Li}(\beta)$.

2. Compute MLEs for both $\alpha$ and $\beta$ as given in (6).

3. Compute $T$ using (5).

4. Repeat the steps $1-3$ for $R^{\prime}$ times and count the number of negative T's.

An estimate of PCS based on simulation can be computed as

$$
\widehat{P C S}=\frac{\text { Number of negative } T^{\prime} s}{R^{\prime}} .
$$

Here $R^{\prime}$ is taken as 1000 . PCS based on simulation and asymptotic results under case 2 are calculated for different parameters choices namely, $\beta=0.2,0.4,0.8,1,1.5,3,6$ and different samples sizes $n^{\prime}=20,40,60,80,100$ is presented in Table 2.

From Tables 1 and 2, it is clear that PCS based on simulation and asymptotic results do not vary much even for moderate sample sizes.

Table 1 PCS based on simulation and asymptotic results under case 1.

\begin{tabular}{cccccc}
\hline $\boldsymbol{\alpha} \backslash \boldsymbol{n}$ & $\mathbf{2 0}$ & $\mathbf{4 0}$ & $\mathbf{6 0}$ & $\mathbf{8 0}$ & $\mathbf{1 0 0}$ \\
\hline 0.2 & $0.657(0.733)$ & $0.757(0.810)$ & $0.821(0.859)$ & $0.881(0.893)$ & $0.921(0.918)$ \\
0.4 & $0.578(0.672)$ & $0.695(0.735)$ & $0.731(0.780)$ & $0.779(0.813)$ & $0.825(0.840)$ \\
0.8 & $0.509(0.511)$ & $0.550(0.516)$ & $0.653(0.519)$ & $0.658(0.522)$ & $0.733(0.525)$ \\
1 & $0.479(0.592)$ & $0.572(0.630)$ & $0.587(0.657)$ & $0.620(0.680)$ & $0.692(0.700)$ \\
1.5 & $0.454(0.564)$ & $0.506(0.590)$ & $0.507(0.610)$ & $0.569(0.627)$ & $0.591(0.641)$ \\
3 & $0.375(0.530)$ & $0.416(0.542)$ & $0.464(0.552)$ & $0.465(0.560)$ & $0.527(0.567)$ \\
6 & $0.383(0.512)$ & $0.410(0.517)$ & $0.405(0.520)$ & $0.460(0.524)$ & $0.433(0.526)$ \\
\hline
\end{tabular}


Table 2 PCS based on simulation and asymptotic results under case 2.

\begin{tabular}{cccccc}
\hline $\boldsymbol{\beta} \backslash \boldsymbol{n}^{\prime}$ & $\mathbf{2 0}$ & $\mathbf{4 0}$ & $\mathbf{6 0}$ & $\mathbf{8 0}$ & $\mathbf{1 0 0}$ \\
\hline 0.2 & $0.855(0.804)$ & $0.898(0.886)$ & $0.930(0.938)$ & $0.951(0.956)$ & $0.974(0.972)$ \\
0.4 & $0.820(0.740)$ & $0.851(0.815)$ & $0.902(0.864)$ & $0.907(0.898)$ & $0.935(0.922)$ \\
0.8 & $0.740(0.660)$ & $0.771(0.717)$ & $0.809(0.759)$ & $0.840(0.793)$ & $0.831(0.819)$ \\
1 & $0.719(0.630)$ & $0.756(0.684)$ & $0.763(0.722)$ & $0.780(0.751)$ & $0.797(0.776)$ \\
1.5 & $0.670(0.590)$ & $0.683(0.628)$ & $0.727(0.655)$ & $0.732(0.678)$ & $0.735(0.697)$ \\
3 & $0.656(0.540)$ & $0.649(0.557)$ & $0.644(0.569)$ & $0.626(0.580)$ & $0.622(0.589)$ \\
6 & $0.651(0.510)$ & $0.632(0.520)$ & $0.618(0.530)$ & $0.598(0.529)$ & $0.618(0.532)$ \\
\hline
\end{tabular}

\section{REAL LIFE ILLUSTRATION}

In this section, the proposed discrimination procedure is illustrated using two real life data sets.

Data set 1: The data set represents the failure times in days of the air conditioning system of 30 aeroplanes (Linhart and Zucchini [17]). For this data, MLEs are calculated using (6) and respective log likelihoods and $T$ are obtained as

$$
\begin{aligned}
\operatorname{In} L_{\text {exp }}(\hat{\alpha}) & =-132.1914, \\
\operatorname{In} L_{L i}(\hat{\beta}) & =-127.3682, \\
T & =-4.8232 .
\end{aligned}
$$

Since $T<0$, it is more appropriate to use Lindley distribution to model this data. To calculate estimated PCS value, 1000 bootstrap samples are generated from the data and the estimated PCS is obtained as 0.9363 . This means that the chance of selecting the wrong model using the proposed procedure is only 6.37\%. Next, $p$ value for the observed $T$ based on asymptotic results is calculated under case 1 and case 2 . First, exponential distribution is considered as the null distribution. Using (10) and (11), asymptotic mean and variance of $T$ are obtained as 4.4184 and 12.6784 respectively. Using (12), the observed $p$ value is found to be 0.0047 . For case 2 , using (14) and (15), asymptotic mean and variance are obtained as -3.0483 and 62.9556 respectively. Using (16), the observed $p$ value is found to be 0.5885 . It is clear that $p$ values in both the cases favor using Lindley distribution to model the data.

Data set 2: This data set consists of waiting time in minutes of 100 customers in a bank (Ghitany et al. [2]). For this data set, MLEs are calculated using (6) and respective log likelihoods and $T$ are obtained as

$$
\begin{aligned}
\operatorname{In} L_{\text {exp }}(\hat{\alpha}) & =-72.52, \\
\operatorname{In} L_{L i}(\hat{\beta}) & =-71.6543, \\
T & =-0.8745 .
\end{aligned}
$$

Since $T<0$, Lindley distribution can be used to model the data. To calculate PCS value, 1000 bootstrap samples are generated from the data and the estimated PCS is obtained as 0.8009 . This implies that the chance of selecting the wrong model using the proposed procedure is $19.91 \%$.

Now $p$ value for the observed $T$ using asymptotic results is calculated under both the cases. First, exponential distribution is considered as the null distribution. Using (10) and (11) asymptotic mean and variance of $T$ are found to be 2.3021 and 5.8099 respectively. Using (12), the corresponding $p$ value is found to be 0.0937 . For case 2, Lindley distribution is considered as the null distribution and using (14) and (15), the values of asymptotic mean and variance of $T$ are calculated as -1.8359 and 54.3614 respectively. Using (16), $p$ value for the observed $T$ is found to be 0.4481 . From $p$ values, it is clear that Lindley distribution is the better fit to the data.

\section{CALCULATION OF MINIMUM SAMPLE SIZE}

In Section 4, it is found that PCS based on simulation and asymptotic results do not vary much even for moderate sample sizes. Therefore, using the asymptotic results, a method to calculate the minimum sample size required to discriminate between exponential and Lindley distribution for a user specified PCS and threshold level is proposed in this section. The distance between two distributions can be measured using Kolmogorov-Smirnov distance, Hellinger distance, etc. It is intuitive that if two distributions are very close (far away), large (small) sample is required to discriminate between them. Thus, in practice, it is of interest to find the minimum sample size required to discriminate between two distributions for a user specified PCS and threshold level based on some distance measure. 
The procedure for finding minimum sample size required to discriminate between the two distributions under case 1 is given as follows. Assume the threshold level to be $D^{*}$ and a PCS of at least $p^{*}$. Using (12), we get

$$
\begin{aligned}
& 1-\Phi\left(-\frac{E_{\text {exp }}(T)}{\sqrt{V_{\text {exp }}(T)}}\right)>=p^{*} \\
\Rightarrow & \left(-\frac{n E_{\text {exp }}(T) / n}{\sqrt{n V_{\text {exp }}(T) / n}}\right)>=1-p^{*} .
\end{aligned}
$$

Taking $\Phi^{-1}$ on both the sides, squaring and rearranging the terms, the minimum sample size $\left(n^{*}\right)$ is obtained as

$$
n^{*}=\frac{\left[\Phi^{-1}\left(1-p^{*}\right)\right]^{2} V_{\exp }(T) / n}{\left(E_{\exp }(T) / n\right)^{2}}
$$

where $\Phi^{-1}$ is the quantile function of the standard normal distribution and $E_{\text {exp }}(T) / n$ and $V_{\text {exp }}(T) / n$ are obtained from (10) and (11) respectively.

In a similar manner, minimum sample size $n^{\prime *}$ required under case 2 is obtained as

$$
n^{*}=\frac{\left[\Phi^{-1}\left(p^{*}\right)\right]^{2} V_{L i}(T) / n}{\left(E_{L i}(T) / n\right)^{2}}
$$

where $E_{L i}(T) / n$ and $V_{L i}(T) / n$ correspond to (14) and (15) respectively. As a numerical illustration, minimum sample size required to discriminate between exponential and Lindley distributions under both cases is calculated for PCS $p^{*}=0.75$ and at different choices of parameter values and is given in Tables 3 and 4 . In both the cases, Hellinger distance is used as the distance measure.

Suppose a practitioner wants to discriminate between exponential and Lindley distributions for a user specified threshold level, say $D^{*}=0.04$ of the Hellinger distance and PCS $p^{*}=0.75$. From Table 3, it can be seen that Hellinger distance is greater than 0.04 if $\alpha<1$. Therefore, if the null distribution is exponential, then for tolerance level $D^{*}=0.04$, one needs to draw a minimum sample of size $n^{*}=90$ in order to meet the PCS level $p^{*}=0.75$. Similarly, from Table 4 , it can be observed that Hellinger distance is greater than 0.04 if $\beta \leq 1$. Therefore, if the null distribution is Lindley, then for tolerance level $D^{*}=0.04$, the user needs to draw a minimum sample of size $n^{*}=117$ in order to meet the PCS level $p^{*}=0.75$.

\section{CONCLUSION}

In this paper, a discrimination procedure is developed using RML to discriminate between exponential and Lindley distributions based on a given random sample of observations. Also, the asymptotic distribution of the logarithm of the RML statistic is derived and asymptotic expression for PCS is obtained. From simulation study and real life application, it is found that even for moderate sample size the discrimination procedure works quite well. Subsequently, a procedure to calculate minimum sample size required to discriminate between the distributions for given PCS value and threshold level is developed using the asymptotic results. From numerical results, it is observed that for large parameter values, Hellinger distance between the two distributions decreases, thereby requiring more samples to discriminate between them.

Table 3 Minimum sample size under case 1.

\begin{tabular}{lccccccc}
\hline$\alpha$ & 0.2 & 0.4 & 0.8 & 1 & 1.5 & 3 & 6 \\
$n^{*}$ & 23 & 27 & 90 & 145 & 345 & 1574 & 9971 \\
Hellinger distance & 0.103 & 0.0732 & 0.0457 & 0.038 & 0.0262 & 0.0121 & 0.0047 \\
\hline
\end{tabular}

Table 4 Minimum sample size under case 2.

\begin{tabular}{lccccccc}
\hline$\beta$ & 0.2 & 0.4 & 0.8 & 1 & 1.5 & 3 & 6 \\
$n^{\prime *}$ & 18 & 33 & 81 & 117 & 252 & 1306 & 9860 \\
Hellinger distance & 0.1272 & 0.0965 & 0.0627 & 0.0525 & 0.0359 & 0.0159 & 0.0058 \\
\hline
\end{tabular}




\section{CONFLICT OF INTEREST}

The authors declare no conflict of interest.

\section{ACKNOWLEDGMENTS}

The authors thank the editor and anonymous referees for their useful suggestions and comments that helped in improving the article.

\section{REFERENCES}

1. D.V. Lindley, J. R. Stat. Soc. Ser. B Methodol. 20 (1958), 102-107.

2. M. Ghitany, B. Atieh, S. Nadarajah, Math. Comput. Simul. 78 (2008), 493-506.

3. H. Zakerzadeh, A. Dolati, J. Math. Ext. (2009), 313-25.

4. S. Nadarajah, H.S. Bakouch, R. Tahmasbi, Sankhya B. 73 (2011), 331-359.

5. H.S. Bakouch, B.M. Al-Zahrani, A.A. Al-Shomrani, V.A. Marchi, F. Louzada, J. Korean Stat. Soc. 41 (2012), 75-85.

6. V.S. Vaidyanathan, A. Sharon, Stat. Optim. Inf. Comput. 4 (2016), 132-146.

7. H. Krishna, K. Kumar, Math. Comput. Simul. 82 (2011), 281-294.

8. J. Mazucheli, J.A. Achcar, Comput. Meth. Prog. Biomed. 104 (2011), 188-192.

9. D. Al-Mutairi, M. Ghitany, D. Kundu, Commun. Stat. Theory Methods. 42 (2013), 1443-1463.

10. D.R. Cox, in Proceedings of the Fourth Berkeley Symposium on Mathematical Statistics and Probability, California, 1961, vol. 2, $105-123$.

11. D.R. Cox, J. R. Stat. Soc. Ser. B Methodol. 24 (1962), 406-424.

12. R. Dumonceaux, C.E. Antle, Technometrics. 15 (1973), 923-926.

13. D. Kundu, A. Manglick, Nav. Res. Logist. 51 (2004), 893-905.

14. D. Kundu, R.D. Gupta, A. Manglick, J. Stat. Plann. Infer. 127 (2005), 213-227.

15. C. Radhakrishna, Linear Statistical Inference and Its Applications, Wiley, New York, 1965.

16. R. Bartle, The Elements of Integration and Lebesgue Measure, Wiley, New York, 1995.

17. H. Linhart, W. Zucchini, Model Selection, Wiley, New York, 1986. 